\title{
Brainmerge: a Semiotic-Oriented Software Development Process for Intelligence Augmentation Systems
}

\author{
André Luis Ogando Paraense ${ }^{12}$ \\ paraense@dca.fee.unicamp.br \\ andre@cflex.com.br \\ Phone. +55 (19) 3251-5211 \\ ${ }^{1}$ DCA- FEEC - UNICAMP \\ Av. Albert Einstein 400 \\ 13083-852 Campinas - SP, Brazil
}

\author{
Ricardo Ribeiro Gudwin ${ }^{1}$ \\ gudwin@dca.fee.unicamp.br \\ Phone: +55(19) 3788-3819
}

\author{
Rodrigo de Almeida Gonçalves ${ }^{2}$ \\ rodrigo@cflex.com.br \\ Phone: $+55(19) 32515211$
}

\author{
${ }^{2}$ CFlex Labs \\ Av. José Bonifácio 717 \\ 13091-140 Campinas - SP, Brazil
}

\begin{abstract}
In this paper we present "Brainmerge", a software development process designed according to semiotic principles in order to coordinate the steps required for the requirements specification, analysis and design of a special kind of intelligent system, which is usually described as Intelligence Augmentation Systems (IAS). IAS represent an extension of Decision Support Systems (DSS) with the use of AI techniques and usually dynamic decision making processes. Traditional software methodologies are usually inefficient when dealing with IAS, and our methodology aims at fulfilling these shortcomings. To illustrate the methodology we describe a real application in planning the circulation of trains in a single track railroad.
\end{abstract}

\section{InTRODUCTION}

Intelligence Augmentation Systems, a special kind of intelligent systems, which have their genesis in Decision Support Systems [1], are computational systems performing some sort of intelligent decision making based on the cooperation provided by an ongoing dialogue between a human user and a computer system. The main result is that the final decision making is not provided neither by the human being nor by the computer by themselves, but is an evolutionary offspring of the collaboration of the two of them. This cooperation results in the augmentation of human intelligence by means of a computational processing power applied to specific points in the human thought process which suffer from some sort of flaw or inefficiency. Usually, traditional software development processes (using traditional techniques like "use cases") are not suitable for the construction of such a kind of system, because they do not consider how decision making is achieved, in terms of human thought processes and how a computer system may help in improving the intelligence of such decision making. This is the interesting differential of our proposal. IAS are suitable to be applied in dynamic decision making environments, where an optimization system cannot do the job of finding a solution to the problem without the cooperation of human agents in the loop. The motivation for developing a process for constructing IAS comes from the fact that it is very difficult to build software systems that have success in being applied to these dynamic environments that represent most real world problems.

Intelligence augmentation (IA) [2] arises when some of the cognitive tasks that would be performed by a human being are performed by an artificial system, in order to improve the efficiency and effectiveness of that specific process, leaving the human free to concentrate on other parts of the process and therefore augmenting his intellect. According to [3], the two basic principles of IA are based on the Peircean theory of signs, as follows:

- 1 - All thought is materially embodied

- 2 - All thought is dialogical

The first one is based on Peirce's dictum that "all thought is in sign", meaning that knowledge can be exosomatically embodied, i.e., that thoughts can occur outside of a brain or mind, in a computer system, for instance. This statement is the base to explain how IAS can be built to perform some of the cognitive tasks in decision making and therefore augment human intelligence. The second one shows the importance of the ongoing dialogue between human and the computer system, that is, IAS need a human in the loop. IAS are complementary to Artificial Intelligence (AI) systems, which try to solve problems substituting a human being and acting just like him, without the human in the loop.

In this work, we propose the concept of "brainware". A "brainware" is a kind of software which performs a "simile" of a mental process which would be helpful for a human being in the process of intelligent decision making. Examples of "brainware" may include techniques like fuzzy systems, neural networks, evolutionary computation, expert systems and other kinds of intelligent systems whether they are applied in order to reproduce some sort of cognitive task.

Brainwares constitute the core of IAS. The cooperation observed in IAS results in the augmentation of human intelligence by means of a computational processing power applied to specific points in the human thought process which suffer from some sort of flaw or inefficiency. To detect and treat the required brainware points within an IAS, we developed "Brainmerge", a software development process based on a Peircean semiotics theory on how 
thoughts are performed.

\section{BRAINMERGE: CONCEPTS AND FOUNDATION}

Brainmerge was developed to coordinate the specification of IAS requirements and architecture, being complemented by traditional development processes and advanced techniques of assisted operation and support/evolution. Traditional techniques such as use cases and UML diagrams are not thrown away: they are still necessary, but not sufficient.

In Figure 1, we show a diagram with all the steps of the methodology. The first step of software engineering is the construction of a domain model. The objective of such task is to represent the main concepts of the problem to be attacked: well understanding the problem without external influences is a priority. This conceptual model will represent the main processes and the context where the system will act, determining the scope and boundaries between the system to be developed and the processes that already exist.

In the case of IAS, this approach is necessary but not sufficient. After mapping the operational workflow (a task similar to the construction of a traditional business model), there is a need to represent how decision making is achieved in terms of human thought processes. In Brainmerge, this task is accomplished by drawing a special kind of diagram: the cognitive flow diagram (CFD).

The CFD is a special kind of diagram developed to model human cognitive processes in searching for a solution to the problem focused. The objectives are first to understand and model the right sequence of cognitive activities and second to find among the activities represented the ones that may be performed by the IAS. The activities to be realized by a brainware will be marked as points of intelligence augmentation later. The system will work in these points, performing the action and dialoguing with the user to provide the information needed in that point of the process to keep going towards the solution. The CFD takes advantage of semiotic concepts in order to represent the cognitive activities and classify them. A CFD is a representation of the thought flow of a human during the resolution of a problem. The concept of sign as defined by Peirce is central here, as the basic units of thought (knowledge units) in the diagram are Peircean signs.

To decide the points of intelligence augmentation where the system will work, the methodology prescribes the construction of a CFD. This diagram is one of the original differentials of Brainmerge.

The visual elements of the CFD are alike the ones used in an UML activity diagram, but with a different connotation. In Figure 2, we detail the main elements in a CFD.

According to Peirce's theory, there are three categories on which cognitive tasks can be classified:

- Monadic tasks (firstness): aleatory tasks. The generation of the first population of solutions in a GA is a monadic task. A random choice between many alternatives is also put in this set.

- Diadic tasks (secondness): deterministic (mechanical) tasks. They consider only present information, without any estimation of future states. These can be efficient decision making processes, but are based on specific rules to be simply followed. The main activity performed by a case based reasoning system or an artificial neural network is diadic.

- Triadic tasks (thirdness): tasks in which future state estimation is used, usually based on the formulation of a goal, plan or prediction to be achieved. These are called intelligent tasks (following Peirce). Optimization algorithms such as graph search or tabu search represent triadic processes. When a human choose between many alternatives based on a goal state, he or she is performing a triadic cognitive task.

After classifying the cognitive tasks in monadic, diadic and triadic, the points of the cognitive process where intelligence augmentation is needed will be defined. These will be the points where the system being developed will work, exchanging the human cognition processes by the work of a brainware, in a dialogue with the user. This is a design exercise, a very important one in the process of specification of an IAS.

Generally, the points of intelligence augmentation are chosen in the regions of the triadic activities. It is possible that more than one point needs to be attacked, what will give rise to multi-core IAS, having more than one brainware being applied. It is also possible that this design process lead us to the conclusion that there exist no point of intelligence augmentation needed, what would mean that there is no need for an IAS in that specific process.

The explanation of all the steps of Brainmerge would require more space than is available here. So, we opted to explain the main steps using a case study as an example, given in section 3. A more detailed account of the methodology will be addressed in a future publication.

\section{Case study - The Trains project}

Brainmerge was applied to build an IAS to solve the problem of planning the circulation of trains in railroads. The project and development have been held since 2004 at CFlex, a Brazilian business intelligence company, and gave birth to a commercial system called Trains. Nowadays, the system is operating in the most important railroads and logistic companies in Brazil and presented great results. Operating since march 2006 in the biggest logistic company in Brazil, it reduced $16.8 \%$ in the time the trains remained stopped in the central zone of the railroad.

The optimization of resources in a railroad is related to a number of different tactical and operational problems from which planning the circulation is one of the most relevant. In Brazil, most railroads are made of single lines, where only one train can circulate each time. This way, the results in time of circulation are directly related to how well the circulation conflicts (crossings) are solved, along with how the trains are dimensioned (size, weight and speed) and how money is invested in actives. As the tracks are single, the decision of letting one train go and the others wait (train dispatching) can be taken when the trains reach a railroad yard. There is a need to plan future movements of the trains 


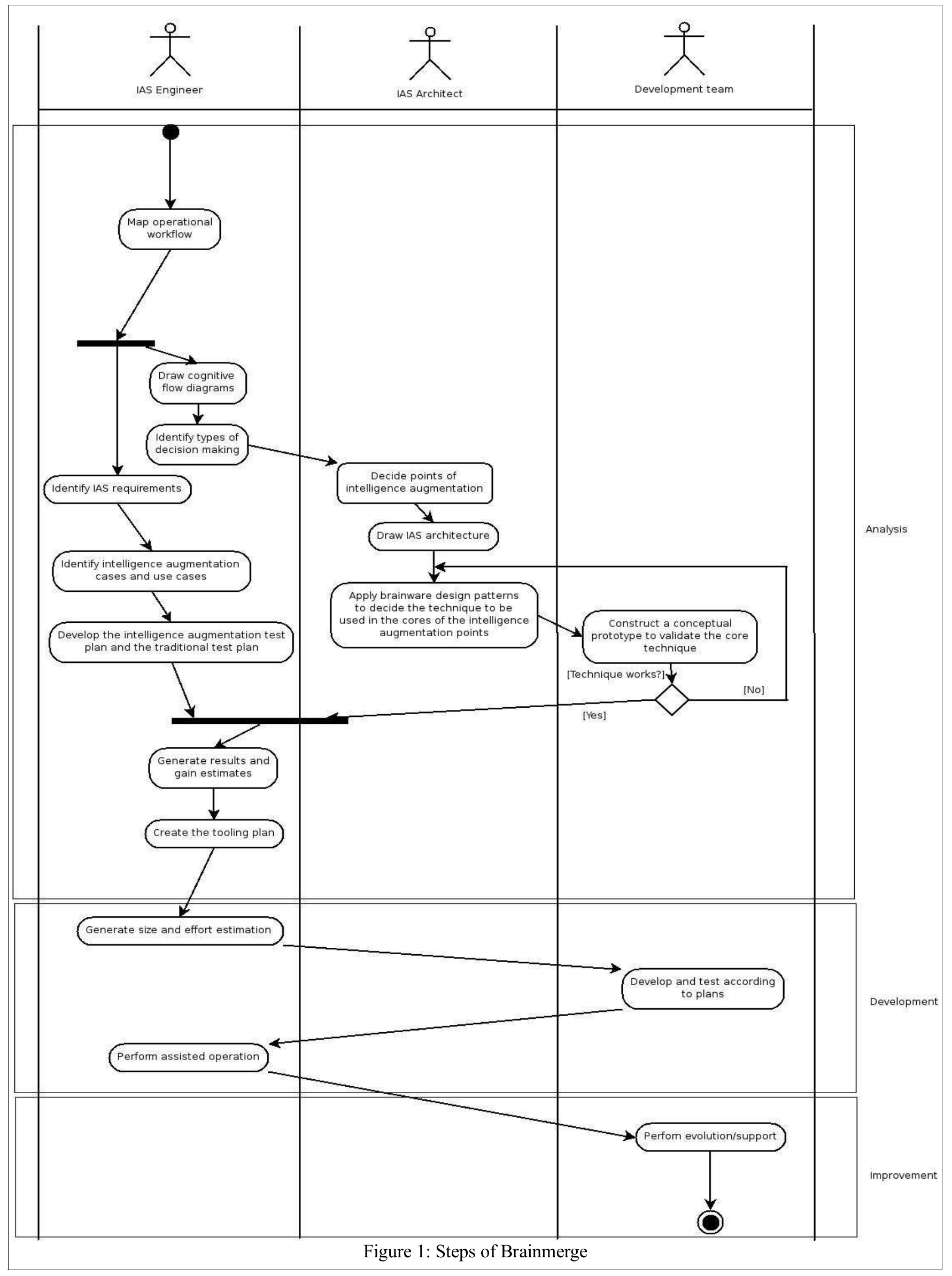


and dispatch them along the railroad in other to prevent blocking, reduce delay time, fuel waste, conflicts in the route of different trains and respect time arrivals, among other issues that are specific for each railroad. This is done by the trains dispatchers from the centralized traffic control (CTC), a centralized control unit from where they align track switches anywhere on the territory so that trains can move into and out of sidings. Also satellite radios and phones enable train dispatchers to communicate directly with train and engine crews.

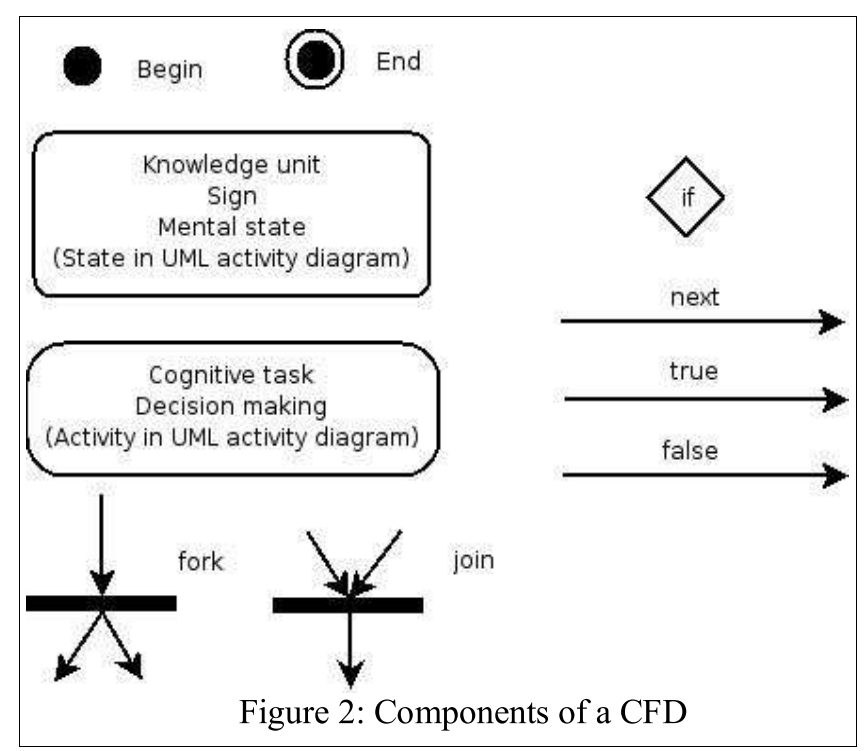

Human train dispatchers need to be familiar with the physical characteristics of the railroad for which they are responsible, as well as the operating capabilities of the locomotive power being used. Experienced train dispatchers learn technical information from locomotive engineers and train conductors and mix that knowledge into the operating decisions made. In some situations, where the decision possibilities are not numerous, an efficient train dispatcher can move a large number of trains over with minimal delay to any train, even in single-track railroad. But this is not true for most of the real situations, where a large number of trains circulate in many control zones composed of a large number of tracks and yards. There are too many problems and restriction that have to be considered by human expert agents solving the problem, and they face many difficulties in performing the job, as the problem becomes combinatorial in size. In spite of their experience of many years, the solutions found are far away from a good one.

To plan the circulation, the planner receives the position of the trains, the route the trains already realized until that moment, the train's activities plan (a series of activities that the train has to perform from its source to its destination with time limits) and a representation of the railroad in a sheet of paper, so the realized can be drawn and the future circulation can be planned. Using a sheet of paper, pencil, eraser and pen, they draw what was realized already by the trains in the railroad until that moment with a pen. With the pencil, the planner start to plan the next movements for the subsequent hours of circulation.

No optimization algorithm is capable of finding the optimal solution to be used in real time by the operation of the railroad. Some alternative approaches to the problem try to find the best or a good solution considering only a few variables that are critical, like the crossing time. However, this is considered to be a naive approach, as the complex environment of the railroad, its operation, points of loading and other agents (like the conductors) are dismissed. It is worth remembering that real problems involve mandatory restrictions and many decision variables.

Some people are tendentious to believe that the changes inherent to the dynamical environment of the railroad will give rise to new optimization problems, and all that is needed is to re-enter the inputs and optimize again in a classical optimization system. This is a huge mistake that has caused the failure of many approaches to problems like this one. Unfortunately, it is not in the scope of this work to prove it with a real experiment.

What is needed in this case is an IAS capable of augmenting human intellect in the points where the activities towards a solution are inefficient and providing a way for the team formed by the planner, the dispatcher and the IAS to find a solution as good as they can in real time for the railroad, facilitating the communication between the parts involved, providing optimization answers and relevant data in the right time, dealing with imprecise information and negotiating the solutions with other agents. In other words, even though the IAS will use optimization algorithms and soft computing techniques, he will be meant to be a tool of intelligence augmentation of the planner, instead of trying to put everything inside an optimization system which will substitute the planner and find a solution intending to be optimal to the problem by itself. In order for the system to do so, it would have to embody a cognitive capacity similar to all human agents that participate in the process and also have access to the information they have, what seems to be very hard, not to say impossible.

After mapping the operational workflow and constructing a domain model for the problem, a CFD was developed in order to complement the understanding of the problem in terms of human thought processes (see figure 3).

The next step of the methodology is to classify the activities in monadic, diadic or triadic cognitive tasks. They are represented respectively by the letters $M, D$ and $T$ in the diagram in Figure 3.

After classifying the activities, the points of intelligence augmentation are marked. In this case, one big spot was chosen. The idea is to develop a brainware which will be able to perform these task and suggest a solution to these activities that will be confronted and negotiated with the solution provided by the human planners. By doing this, Trains will not only provide a media of representation for all other activities and for the planner to represent his solutions, what corresponds to a way to represent the knowledge units in the CFD, but also perform some of the activities and suggest a solution, augmenting human intellect. As expected, the point was marked around the triadic activities.

The main IAS attributes were considered as follows in the development of Trains requirements. The media of representation permits to create, visualize and edit the plan 
of circulation. The interface is made of a graphic representation of the railroad (train's graph), a control panel, dialogue boxes and some other peripheral interfaces.

The trains' graph is an example of success as a media of representation. It is able to represent all signs needed for the planner or the IAS to represent a solution. Most of these symbols are represented as icons, facilitating to understand the solution proposed and to manipulate it with no impedance. Complex knowledge units making part of the railroad at the same time in different tabs. This feature gave more power to the cognitive flow, as the planner is able to have a global understanding of the railroad and plan each control zone. The user can criticize the solution provided with mouse clicks, changing movements and decisions proposed.

The attribute of teleology is of great importance for the success of Trains. The capacity of the system to suggest a

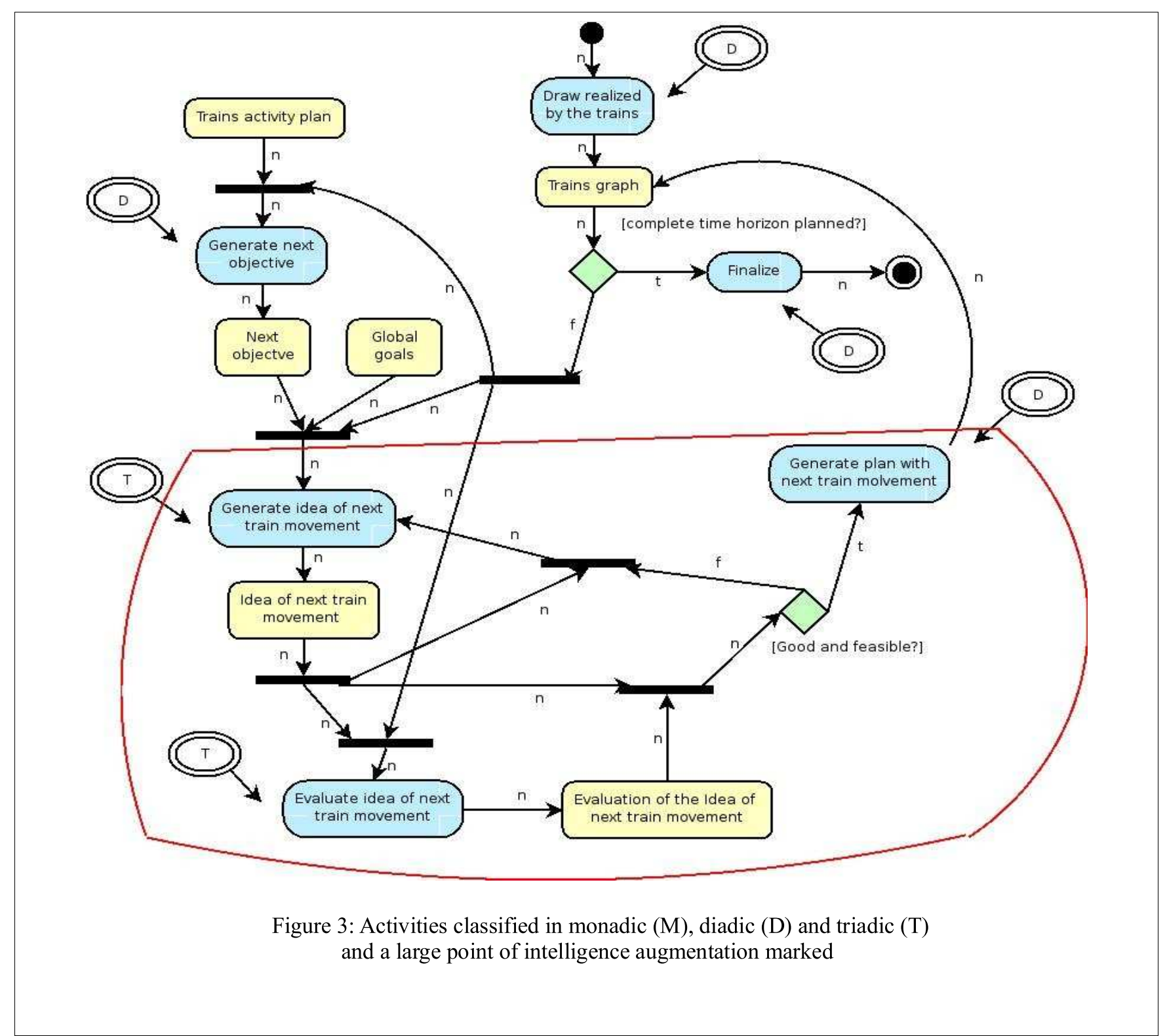

solution can be edited as simple objects dragged with the mouse, as the IAS performs all the calculations and modifications in the background. The train's graph is more than a tool where movements are represented and planned: it can be seen as a special language through which the solution is represented and the dialogue with the user takes place. It is through the trains graph that the system will suggest its solutions found by the brainware and the user will criticize them and suggest other solutions. It can be seen in Figure 4.

Moreover, Trains is able to represent all control zones of the good solution in real time operation, taking only a few seconds and respecting all restrictions present in the problem, make it possible for the user to evaluate it according to some knowledge outside the system and criticize it, quickly changing some characteristics as the media of representation allows to do so. There is even the possibility to try different ideas, simulating diverse scenarios and according to the results choosing the one that fits best in operational time.

Planning the circulation is multi-objective and time 


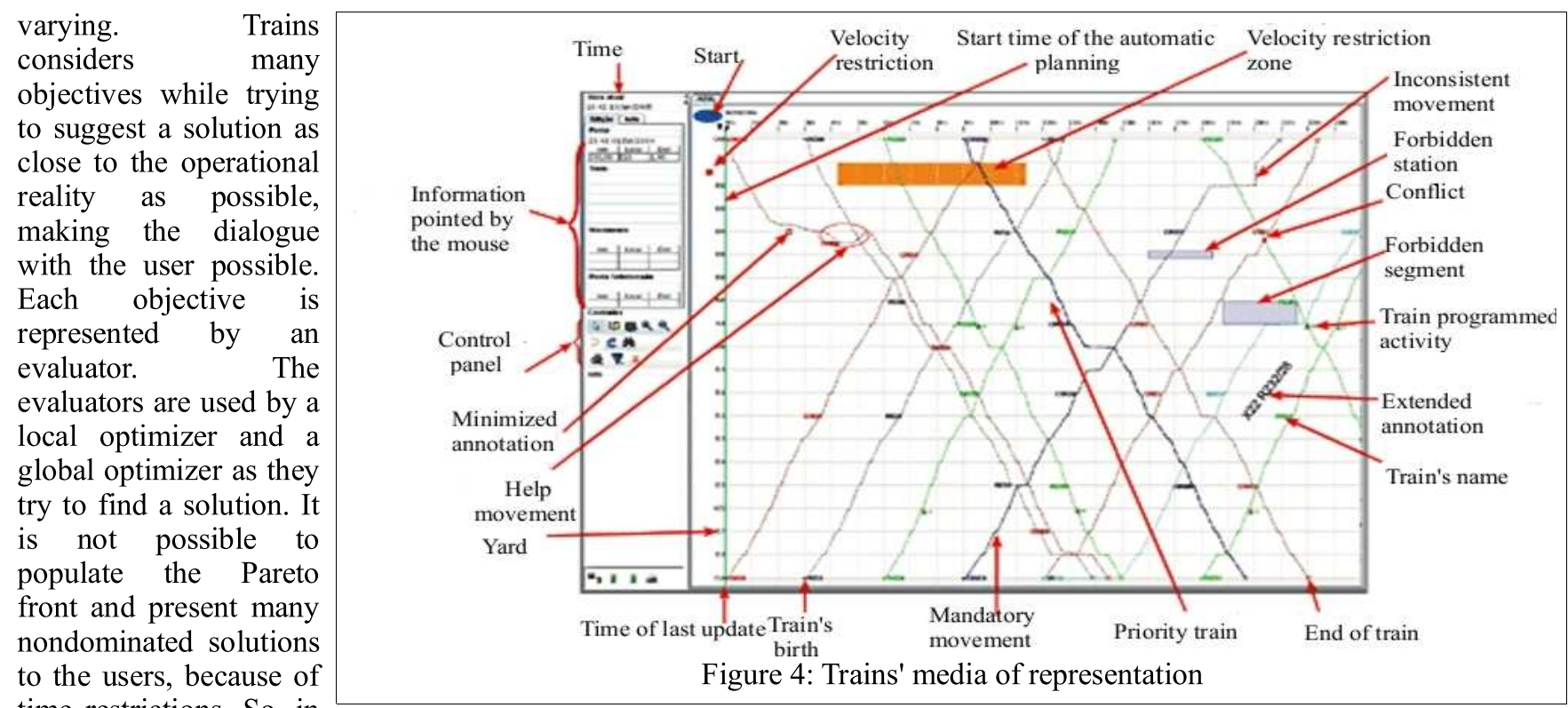
time restrictions. So, in

this case, Trains ponderates the objectives according to operational scenarios pre-configured by the user, who can create new scenarios. Some objectives are common to many railroads, like reduce crossing time or saving fuel, but in the end of the month, sometimes it is more interesting to meet the goals of transportation than care about fuel savings. As the solution is reached, the system provides a collection of statistics and reports on the details relative to the objectives pondered.

The system has a politic of evolution instead of features implemented. Each railroad is a different case, as objectives, models and restrictions are different, and the system needs to be customized. This way, Trains is constantly growing as it is installed in different railroads.

The reason for Trains to be successful in solving the circulation planning problem, which is a dynamic real time problem with many restrictions, is that it was developed as an IAS, instead of a simple optimization system trying to find an optimal solution to the problem. Instead of trying to find this optimal plan, it provides a way to the team formed by the planners, dispatchers and Trains itself of finding a very good solution in real operational time, each one with different cognitive capacities. The role of Trains in this case is to work more in the points where the human brain presents a flaw or inefficiency so the rest of the team can work better in other parts of the problem. Trains is a multiagent system integrating brainwares (computational agents) and real brains (human agents) in the seek for a solution to the circulation planning problem, providing a media of representation where the user represents the solution with no impedance and suggesting a solution in a few seconds to keep the dialogue with the planner and control the cognitive flow of the team.

\section{Conclusions}

Brainmerge is an original software development process capable of overcoming the deficiencies of traditional software development processes in the development of a Intelligence Augmentation Systems. This achievement brings about the possibility of building successful IAS, such as the Trains project presented in the case study. Traditional steps of development like use cases are not abandoned, they are still used and complemented by the steps of Brainmerge, configuring an expansion of more traditional software processes.

The development of a methodology to build IAS brings maturity and trust to the field, being an important motivation to attack real applications and achieve gains in many sectors of the economy, in operational, tactical and strategical levels.

We believe that the semiotic-oriented framework used to model how decision making is achieved, in terms of human thought processes, represents a new paradigm for software development processes suitable for the specification of a system that aims to augment human intellect performing some cognitive tasks. The development of the concept of brainware was very relevant in this sense. The CFD was another important development in this direction.

\section{REFERENCES}

[1] - Shim, J.P., et al. "Past, present and future of decision support technology". Elsevier, Decision Support Systems 33 (2002) 111-126.

\section{[2] - Engelbart, D. C. - "AUGMENTING HUMAN}

INTELLECT: A Conceptual Framework", Summary report. http://sloan.stanford.edu/mousesite/EngelbartPapers/B5 F18 Conce ptFrameworkInd.html

[3] - Ransdell, Joseph - "The Relevance of Peircean Semiotic to Computational Intelligence Augmentation". SEED Journal (Semiotics, Evolution, Energy, and Development), Volume 3, No. 3, 2003 http://www.library.utoronto.ca/see/SEED/Vol3-3/Ransdell.htm 